Methods TV positive samples (by NAAT) were collected from two Public Health Laboratories in the Netherlands, from 2012 to 2016. TV was typed using multi-locus sequence typing (MLST) of 7 household genes. MLST profiles were put into a minimum spanning tree together with reference strains and so allocated a genotype. TVV RNA was detected using nested reverse-transcriptase PCR with newly designed primers and visualised on gel.

Results We included 157 clinical samples from 151 clients (most were female; $n=133,87 \%$ ). In total, 120 samples were genotyped for TV; TV genotype I was most common $(n=65 ; 54 \%)$. Over half of these samples were found positive for TVV RNA $(n=65 ; 54 \%)$. Most TVV infections were found in TV genotype I $(\mathrm{n}=49 ; 75 \%)$. The amplified products for TVV1, TVV2 and TVV3 were respectively $97 \mathrm{bp}, 290 \mathrm{bp}$ and $156 \mathrm{bp}$ and sequencing showed high identity with TVV genomes; TVV1-C344 (93\%), TVV2-OC3 (89\%) and TVV3UR1 $(85 \%)$, supporting specificity of the primers for these targets. TVV1 was most prevalent $(n=45 ; 41 \%)$, followed by TVV3 $(n=34 ; 31 \%)$ and finally TVV2 $(n=31 ; 28 \%)$. Co-infections occurred in $60 \%$ of TVV positive samples. TVV4 was not found at all. Sensitivity for TVV detection still needs to be established using TV samples with a known TVV (genotype) status.

Conclusion TVV detection can be performed directly on clinical samples using nested RT-PCR. TVV infection occurs most frequently in TV type I parasites as in agreement with previous findings. This opens the way for molecular epidemiological studies to gain insight into the role of TVV in TV pathogenicity.

\section{P3.40 SOCIO DEMOGRAPHIC AND SEXUAL BEHAVIOURAL CORRELATES OF HERPES SIMPLEX VIRUS TYPE 2 INFECTION AMONG PREGNANT WOMEN IN SOUTHWESTERN NIGERIA}

${ }^{1}$ Chinenye Gloria Anaedobe, ${ }^{2}$ Fowotade Adeola, ${ }^{3}$ Bakare Rasheed Ajani. 1. University of Abuja, Federal Capital Territory, Abuja, Nigeria; ${ }^{2}$ University College Hospital, Ibadan, Ibadan, Nigeria; ${ }^{3}$ University of Ibadan, Ibadan, Nigeria

\subsection{6/sextrans-2017-053264.277}

Introduction Herpes Simplex Virus type 2 (HSV 2) is the most common cause of genital ulcer disease and facilitates the acquisition of Human Immunodeficiency Virus (HIV). It leads to lifelong latent infection and this raises concerns among women of reproductive age, considering the risk of neonatal transmission. This study aims to determine HSV-2 seroprevalence among pregnant women, identify the correlation with socio-demographic characteristics, sexual behaviour, pregnancy outcomes and co-infection with HIV.

Methods A total of 270 pregnant women attending antenatal clinic of the University College Hospital Ibadan were enrolled in this cross-sectional study. Their serum samples were tested for HSV-2 IgG using type specific third generation ELISA and HIV1, using Uni-Gold Recombigen and ALERE determine. Pretested questionnaire were used to obtain bio-data on sociodemographic characteristics, sexual behaviour and obstetrics history of the participants. Data analyses was done using SPSS version 20 .

Results The seroprevalence of HSV 2 type specific IgG was $33.3 \%(90 / 270)$ and a HIV/HSV 2 co-infection rate of $39 \%$ (35/90) was observed. Logistic regression analysis showed that polygamy, low educational level, positive HIV status, previous sexually transmitted infections (STIs), early age at sexual debut and multiple sexual partners were independent risk factors for HSV-2 infection. Obstetrics complications such as intrauterine foetal death, congenital malformations and spontaneous abortion were predictors of HSV-2 infection.

Conclusion The seroprevalence of HSV-2 in this pregnant population is lower than what is observed in some other SubSaharan African countries; however, co-infection with HIV is high and majority of the women are still susceptible to primary HSV-2 infection in pregnancy. The demographic, sexual behaviour and bad obstetrics histories found to be predictors of HSV 2 infection in this study may be important in selecting candidates for screening tests, developing strategies towards effective health promotion campaign and reducing risk of HIV transmission.

\section{P3.41 PREVALENCE, SOCIO-DEMOGRAPHIC AND SEXUAL BEHAVIOURAL RISK FACTORS FOR HEPATITIS B VIRUS INFECTION AMONG PREGNANT WOMEN IN SOUTHWESTERN NIGERIA}

${ }^{1}$ Chinenye Gloria Anaedobe, ${ }^{2}$ Fowotade Adeola, ${ }^{3}$ Chukwuma E Omoruyi, ${ }^{4}$ Rasheed Ajani Bakare. 'University of Abuja, Federal Capital Territory, Abuja, Nigeria; ' University College Hospital, Ibadan, Ibadan, Nigeria; '3niversity College Hospital Ibadan, Ibadan, Nigeria; ${ }^{4}$ University of Ibadan, Ibadan, Nigeria

\subsection{6/sextrans-2017-053264.278}

Introduction In endemic countries, Hepatitis B virus (HBV) infection occurs mainly during infancy and early childhood, with vertical transmission from an infected mother being a major route of infection. This study aimed at identifying the prevalence and sexual behavioural risk factors for HBV infection among pregnant women in Ibadan, Nigeria.

Methods A cross-sectional study was done at the antenatal clinic of the University College Hospital Ibadan. One hundred and eighty pregnant women were recruited over a six month period, and their serum was tested for Hepatitis B surface antigen (HBsAg) (BIORAD FRANCE) using third generation ELISA and HIV-1 and 2, using Uni-Gold Recombigen and ALERE determine. Positive HBsAg samples were tested for Hepatitis B envelope antigen ( $\mathrm{HBeAg})$, antibody ( $\mathrm{HBeAb}$ ) and Hepatitis B core antibody (HBcAb) (DIAPRO Italy) while serum HBV DNA was detected using PCR. Data were obtained using questionnaires and analysed using SPSS-20

Results The seroprevalence of HBsAg was $8.3 \%$ out of which 26.7\% were positive for $\mathrm{HBeAg}, 53.3 \%$ had $\mathrm{HBeAb}, 20 \%$ had neither $\mathrm{HBeAg}$ nor $\mathrm{HBeAb}, 100 \%$ had total $\mathrm{HBcAb}$ and $86.7 \%$ had HBV DNA in their serum. A co-infection rate (HBV/HIV) of $26.7 \%$ was observed. The mean age was 32.1 years, the highest HBV infection rate occurred in 25-29 year age group. Multiple sexual partners $(\mathrm{OR}-3.987$, P- value $=0.026)$, early age at sexual debut $(\mathrm{OR}-11.996$, $\mathrm{P}$ - value $=0.022)$ were independent risk factors for HBV infection.

Conclusion The result of this study brings to light the high prevalence and high infectivity rate of chronic HBV virus infection among women of child bearing age in Nigeria. Thus, there is a dire need for routine screening for all pregnant women during antenatal care, immunoprophylaxis for exposed newborns and surveillance for those with chronic infection. The socio-demographic and risky sexual behavioural characteristics found to be predictors of HBV infection should be considered when developing strategies towards effective sexual 
health promotion campaign and health education programs on prevention and control measures.

\section{P3.42 INCIDENT TRICHOMONAS VAGINALIS IS ASSOCIATED WITH PARTNERSHIP CONCURRENCY: A LONGITUDINAL COHORT STUDY}

C Kenyon, 'J Buyze, ${ }^{1} \mathrm{M}$ Klebanoff, ${ }^{2},{ }^{3} \mathrm{R}$ Brotman ${ }^{4}$. 'Institute of Tropical Medicine, Antwerp, Belgium; ${ }^{2}$ The Ohio State University College of Public Health, USA; ${ }^{3}$ The Research Institute at Nationwide Children's Hospital; ${ }^{4}$ University of Maryland School of Medicine, USA

\section{$10.1136 /$ sextrans-2017-053264.279}

Introduction Sexual partner concurrency has been shown to be a risk factor for a number of sexually transmitted infections but it is unknown if it is a risk factor for Trichomonas vaginalis (TV).

Study design We used mixed effects logistic regression to assess the association between partner concurrency and incident TV in the Longitudinal Study of Vaginal Flora, a cohort study of 3620 women followed quarterly for five visits.

Results TV was more common in those reporting definite/possible/unknown PC $(15.6 \% / 15.0 \% / 18.3 \%)$ than those reporting no PC $(5.2 \%-P<0.001$ for all three comparisons). After controlling for a range of confounders, incident TV remained associated with reporting that one's partner definitely (adjusted Odds ratio [aOR] 5.4; 95\% Confidence Interval [CI], 3.7-8.0) and possibly (aOR 3.4; 95\% CI, 2.2-5.1) engaged in partner concurrency in the preceding time period.

Conclusion Partner concurrency was associated with incident TV infection. We hypothesise that this association may be partly explained by concurrent partnering bypassing a 'rapidclearance-in-males-bottleneck.'

\section{P3.43 MODELLING THE SPREAD OF GONORRHOEA IN AN MSM POPULATION}

${ }^{1} \mathrm{C}$ Kenyon, J Buyze, ${ }^{1} \mathrm{~N}$ Hens, ${ }^{2,3}$. ${ }^{1}$ Institute of Tropical Medicine, Antwerp, Belgium; ${ }^{2}$ University of Antwerp, Belgium; ${ }^{3}$ Hasselt University, Belgium

10.1136/sextrans-2017-053264.280

Introduction There is considerable uncertainty as to the effectiveness and optimal timing of Neisseria gonorrboea (NG) screening in Men who have Sex with Men (MSM). NG has evolved resistance to a wide range of antibiotics, which makes it particularly important to ensure that NG screening in this population does not lead to excessive consumption of antibiotics. We use a mathematical model to evaluate the effectiveness of different NG screening strategies on NG prevalence in an MSM population.

Methods Separable Temporal Exponential Random Graph Models are used to model the sexual relationships network in MSM. Two networks of sexual relationships with main and casual partners are modelled, where the number of casual partners depends on having a main partner or not and vice versa. Next the transmission of NG is simulated on this dynamic network. We have adapted the standard model to include different infection statuses per person for the pharynx, urethra and rectum. Accordingly, different possible transmission routes (anal sex, oral sex and rimming, both active and passive) with their own act and transmission rate have been implemented. Furthermore, a different recovery rate for symptomatic and asymptomatic infections was specified. The model was used to compare different screening programmes in terms of NG prevalence. Our models simulate day-by-day evolution of a population of 10,000 MSM. Each scenario is simulated 20 times for 10 years. Behavioural data was taken from Belgian MSM participating in the European Men who have sex with men Internet Survey (EMIS).

Results If one half of MSM is screened once a year, the prevalence of NG infection at pharynx decreases from $13 \%$ to $10 \%$ (urethral), $8 \%$ to $6 \%$ (rectal) and $16 \%$ to $12 \%$ (pharyngeal), as compared to no screening. When only one third is screened, prevalence decreases to $11 \%, 7 \%$ and $13 \%$, respectively.

Conclusions The achieved prevalence reduction might not outweigh the larger risk of development of antibiotic resistance.

\section{P3.44 JUST GOOGLE IT! THE IMPACT OF MEDIA COVERAGE OF AN OUTBREAK OF HIGH-LEVEL AZITHROMYCIN RESISTANT GONORRHOEA ON ATTENDANCES, TESTING AND DIAGNOSES AT LOCAL SEXUAL HEALTH CLINICS}

${ }^{1} \mathrm{C}$ Smolarchuk, ${ }^{1} \mathrm{M}$ Furegato, ${ }^{1} \mathrm{H}$ Mohammed, ' $\mathrm{K}$ Town, ${ }^{1} \mathrm{H}$ Fifer, $2,3 \mathrm{~J}$ Wilson, ${ }^{1} \mathrm{~A}$ Nardone, and ${ }^{1} \mathrm{G}$ Hughes. ${ }^{1}$ National Infection Service, Public Health UK; ${ }^{2}$ Leeds Teaching Hospitals NHS Trust, UK; ${ }^{3}$ Leeds Sexual Health, UK

\subsection{6/sextrans-2017-053264.281}

Introduction An outbreak of high-level azithromycin resistant gonorrhoea, dubbed 'super gonorrhoea' in the mainstream media, emerged in Leeds, England in 2015 and has since spread to other parts of the country. We aimed to determine if media coverage affected online searches nationally and attendances, and gonorrhoea testing and diagnoses locally at sexual health clinics (SHCs).

Methods Google Trends was used to determine Relative Search Interest (RSI) for 'gonorrh"' and 'super gonorrh"' in England from 2015-2016. Using data from England's national STI surveillance system (GUMCADv2), an interrupted time series analysis was performed to compare the sex-stratified, weekly rates of attendances, and gonorrhoea testing and diagnoses at 6 SHCs in Leeds and in other affected areas. The analysis compared rates of events 6 weeks before and after initial media coverage of the outbreak in September 2015.

Results The RSI peaked during initial media coverage in September 2015 (100) with smaller peaks in December 2015 (47), April 2016 (72), and September 2016 (33), coinciding with subsequent coverage. The number of SHC attendances by women in Leeds rose after initial media coverage $(p<0.01)$ by $36 \%$ (from 320 to $435 /$ week), but there was only a $4 \%$ increase in attendances (from 326 to $340 /$ week) by men $(p=0.70)$. There was no change in rates of gonorrhoea tests or diagnoses in women $(p=0.87$ and 0.23$)$ or men $(p=0.51$ and $p=1.00)$. There were no significant increases in event rates in other areas with a high RSI including Birmingham, Manchester, London, Liverpool and Sheffield.

Conclusion Media coverage of the outbreak was associated temporally with increased online searches for gonorrhoea nationally, and female attendances at SHCs in Leeds only. This demonstrates opportunities for health promotion for the prevention and control of outbreaks and raises the question of how best to target such messaging to those populations and groups most likely to benefit from attending and being tested in SHCs. 